TRANSFORMANDO A MULTIPLICIDADE DE SISTEMAS NUM SISTEMA UNIFICADO: DESAFIOS DA UNIFICAÇÃO NO INT

\title{
TRANSFORMING THE MULTIPLICITY OF QUALITY SYSTEMS INTO ONE: THE INT EFFORT
}

\author{
SIMÕES, Maria Gabriela Pinto de Almeida ${ }^{1 *}$; CLARIM, Haroldo de Jesus²; GAIO, Arnaldo Pinheiro \\ Costa $^{3}$
}

1,3 Instituto Nacional de Tecnologia, Divisão de Gestão da Qualidade, Av. Venezuela, 82, sala 709 cep 200813129, Rio de Janeiro - RJ, Brasil

(fone: +55 212123 1170; fax: +55 2121231284 )

${ }^{2}$ Instituto Nacional de Tecnologia, Coordenação de Gestão Tecnológica, Av. Venezuela, 82, $8^{\circ}$ andar cep 20081-3129, Rio de Janeiro - RJ, Brasil

(fone: +55 212123 1260; fax: +55 2121231284 )

\author{
* Autor correspondente \\ e-mail: gabriela.simoes@int.gov.br
}

Received 12 June 2000; received in revised form 30 November 2000; accepted 14 December 2000

\section{RESUMO}

Em novembro de 2006, o Instituto de Metrologia, Qualidade e Tecnologia - Inmetro, por meio de sua Divisão de Acreditação de Laboratórios - DICLA, enviou ofício as Organizações com Laboratórios Acreditados, sugerindo que as que possuíssem mais de um sistema da qualidade com ensaios acreditados promovessem a sua unificação no sentido de racionalizar as atividades e recursos de acreditação dos laboratórios. O presente trabalho apresenta o esforço traduzido em ações e estratégias adotadas pelo Instituto Nacional de Tecnologia INT, a partir de 2008, visando a implantação de um Sistema de Gestão da Qualidade Unificado, segundo os critérios da ABNT NBR ISO/IEC 17025 o que foi conseguido em 2012, após a reavaliação de todo os laboratórios e mudança para um único escopo acreditado. O INT deixou de ter seis laboratórios acreditados e passou a ter um único escopo, sob o número de acreditação CRL 0006, o primeiro conseguido por um de seus laboratórios em 1984. Esta mudança trouxe como resultado maior conhecimento sobre o Sistema de Gestão da Qualidade, aumentando a capacidade do INT de garantir a qualidade de seus ensaios acreditados.

Palavras-chave: Gestão da Qualidade, Acreditação, ISO/IEC 17025.

\begin{abstract}
In November 2006, the INT received an informative document from the National Institute of Metrology, Quality and Technology - the Brazilian accreditation body - which recommended that institutions with more than one accredited quality management system for laboratory tests should promote its unification. This paper presents the INT effort, through actions and strategies, started in 2008 toward the unified implementation of the Quality Management System due to the ISO/IEC17025 criteria. INT's accreditation (CRL006) was achieved in 2012, after the reassessment of all the laboratories accredited. The INT has transformed six accredited laboratories into just one acredited institution under the number CRL006. This was the first number achieved by an INT'S lab in 1984. This change has brought one particular output such as higher knowledge of the quality management system which makes INT as institution more capable to ensure the quality of all acredited tests.
\end{abstract}

Keywords: Quality management, Accreditation, ISO/IEC 17025. 


\section{INTRODUÇÃO}

O Instituto Nacional de Tecnologia - INT é uma instituição pública da administração direta federal, integrante do Ministério da Ciência, Tecnologia e Inovação. Desde sua criação em 1921, o INT vem participando ativamente com a missão de "Participar do desenvolvimento sustentável do Brasil, por meio da pesquisa tecnológica, da transferência do conhecimento e da promoção da inovação"1 quer seja, na forma de suporte tecnológico às indústrias, na capacitação de recursos humanos ou na geração e disseminação de tecnologias.

No início da década de 90 , foi definido, como estratégia, pela Direção do INT, consolidar e expandir a infraestrutura de serviços tecnológicos para atender ao Programa Tecnologia Industrial Básica - TIB criado pelo Governo Federal, cujo objetivo visava à melhoria da qualidade dos produtos fabricados no País e o aumento das exportações. Para tal, consolidou sua atuação em Normalização e Regulamentação Técnica, iniciando sua atuação em Metrologia e Avaliação da Conformidade com a implantação de sistema de gestão da qualidade nos seus laboratórios segundo os requisitos da norma ABNT ISO Guia 25, atual NBR ISO/IEC 17025.

Por meio de projetos financiados no
âmbito do Programa de Apoio ao Desenvolvimento Científico e Tecnológico PADCT e com recursos próprios, o INT ampliou sua capacitação laboratorial e de recursos humanos, de forma a aumentar o número de ensaios e laboratórios acreditados pela Rede Brasileira de Laboratório de Ensaios - RBLE, bem como acreditar laboratórios na Rede Brasileira de Calibração - RBC, ambas gerenciadas pelo INMETRO.

Ao longo do tempo, o INT buscou outros reconhecimentos em Gestão da Qualidade como a participação em prêmios da Qualidade tais como: do Governo Federal, em 1998; o Programa de Excelência na Gestão voltado para as Entidades de Pesquisas, Desenvolvimento e Inovação, que participa desde 1999 e o Prêmio Qualidade Rio em 2003.

O aumento significativo no número de contratos de acreditação e outros reconhecimentos, bem como do número de

1 Esta missão foi definida para o período 2011-2021 ensaios, é resultado da manutenção bem sucedida de um Sistema de Gestão da Qualidade voltado às demandas da sociedade.

\section{DESENVOLVIMENTO}

O Sistema de Gestão normalmente utilizado por laboratórios de calibração e ensaio é o proposto pela norma ABNT NBR ISO/IEC 17025, que inclui requisitos referentes à direção (requisitos e gestão) e de competência técnica para a realização de calibrações e ensaios.

Segundo SANTOS (2011), instituições que possuem diversos laboratórios podem adotar Sistemas de Gestão da Qualidade individuais ou um Sistema de Gestão da Qualidade único para todos os laboratórios. Quando a instituição adota Sistemas de Gestão da Qualidade individuais, cada laboratório possui sistemáticas próprias para atender os requisitos da norma. No caso de um Sistema de Gestão da Qualidade unificado, contemplando diversos laboratórios, que atendem a um único padrão normativo, há uma Gestão centralizada e uniforme em todos os laboratórios.

Comprometimento e atitude dos colaboradores, aliados ao suporte da Direção e ao trabalho em equipe são fatores críticos recorrentes na implantação do Sistema de gestão. Quando a alta direção não está envolvida e as equipes não estão comprometidas com a adequação e a eficácia do sistema, não há como mantê-lo. A qualidade necessita convergência de esforços em todos os níveis da hierarquia organizacional. No caso do INT, a alta direção define a política e metas institucionais para a qualidade, bem como disponibiliza recursos necessários à implantação e manutenção de um Sistema da Qualidade que atenda aos requisitos da acreditação, bem como os das agências governamentais demandantes de ensaios e calibrações.

Convergindo em direção a essas metas, em 2008, foi iniciado o processo de unificação. Naquele momento, o INT possuía sete Sistemas de Gestão da Qualidade implantados, em sete laboratórios, referentes aos escopos acreditados, consistindo em um sistema retalhado onde não havia interação entre os mesmos. O nível de amadurecimento de cada um dos sistemas também era heterogêneo: existindo sistemas com 20 anos de acreditação em contraposição com outros recém-acreditados. 
A unificação do sistema foi coordenada pela Divisão de Gestão da Qualidade - DIGQ, que é responsável, de acordo com o Regimento Interno do INT, pelas seguintes atividades:

I. Prestar assessoramento superior no âmbito das ações especiais do INT;

II. Exercer a função de secretaria executiva do Comitê Gestor da Qualidade;

III. Orientar a estruturação e organização das atividades do INT no âmbito do sistema da qualidade, estabelecendo e mantendo a documentação correspondente;

IV. Orientar a elaboração e revisão da documentação do sistema da qualidade do INT; e

V. Promover as atividades de desenvolvimento organizacional, processos de meIhorias e auditorias internas da qualidade;

De modo a unificar os sistemas foram elencadas as seguintes ações, por ordem de importância:

- Unificação dos Manuais da Qualidade e escolha de um único Gerente da Qualidade;

- Definição dos Responsáveis Técnicos por escopo e não mais por laboratório;

- Unificação dos Requisitos Gerenciais (item 4) da norma ABNT NBR ISO/IEC 17025:2005;

- Unificação dos Requisitos Técnicos (item 5) da norma ABNT NBR ISO/IEC 17025:2005.

A escolha de iniciar pelo Manual da Qualidade se deve ao fato dos mesmos serem muito parecidos e utilizarem alguns procedimentos comuns a toda à instituição. As acreditações no INT eram tratadas por laboratório, onde a alta direção consistia no chefe da Divisão a qual o laboratório era vinculado, e não havia envolvimento em nível de coordenação e direção da instituição. Com a unificação do manual, verificou-se que esta etapa só apresentava uma dificuldade - a gerência da qualidade não pertenceria mais a somente um laboratório, mas a todo o INT. A partir desta constatação, foi estabelecido através de Portaria do INT, um único Gerente da Qualidade, com a atribuição de atender a todos os laboratórios com ensaios acreditados.

Ainda neste contexto, foi emitida outra Portaria estabelecendo o "Comitê de Análise Crítica do Sistema de Gestão da Qualidade". Esse Comitê, em um primeiro momento, tem a função de analisar criticamente as documentações emitidas pela DIGQ e uniformizar as políticas e os procedimentos, definidos pelo INT, para atender a cada um dos requisitos da norma aplicada. De modo a propiciar a participação e o envolvimento de todos os laboratórios com escopo acreditado e postulantes de acreditação, foram definidos como participantes deste fórum, os Responsáveis Técnicos por escopo, o Gerente da Qualidade, o Chefe da Divisão de Gestão da Qualidade e os respectivos Coordenadores de cada área (alta Direção).

Observando a norma ABNT NBR ISO/IEC 17025 , os requisitos do item 4 (requisitos gerenciais) são documentos relativos a "parte administrativa" das unidades. Neste sentido vale lembrar que como cada unidade era isolada, existiam 7 procedimentos para cada um dos itens deste requisito, isto é, eram aproximadamente 100 procedimentos para atender os requisitos gerenciais, que poderiam ser reduzidos a aproximadamente 15 procedimentos com a unificação.

Os elementos do sistema da qualidade do INT foram estabelecidos segundo a seguinte hierarquia:

Manual da Qualidade - MQ

Normas Gerenciais da Qualidade - NGQ

Procedimentos Operacionais da Qualidade POQ

Registros da Qualidade - REQ

Assim, com a unificação do Manual da Qualidade para todos os laboratórios, ficou definido que todos os requisitos gerenciais seriam atendidos por Normas Gerenciais da Qualidade - NGQs, documentos estes que são únicos para todo o INT. Os Procedimentos Operacionais da Qualidade - POQs descreveriam, então, a metodologia de ensaio adotada e os REQs seriam os registros que permeiam todo o sistema de gestão. Todos estes documentos passaram a ser controlados pela 
Divisão de Gestão da Qualidade e ganharam um modelo e formatação única.

Com a unificação do Manual e percepção de que o processo de acreditação envolveria algumas atividades realizadas fora do laboratório, alguns setores que já desenvolviam atividades ligadas aos requisitos da ABNT NBR ISO/IEC 17025, mas que não estavam envolvidos com o processo de acreditação passaram a ser treinados, qualificados e avaliados em seus requisitos conforme pode ser verificado na Tabela 1.

O processo de aquisição de serviços e insumos; análise crítica de solicitação de clientes, gerenciamento de reclamações e atendimento ao cliente, gerenciamento de trabalhos não conformes, ações corretivas, preventivas e melhorias; auditorias internas e análise crítica pela direção foram os requisitos unificados até esse momento, sempre com o cuidado de atender às necessidades de todas as unidades envolvidas.

Tabela 1 - Setor do INT/Requisitos da ABNT NBR ISO/IEC 17025

\begin{tabular}{c|c}
\hline Setor & Requisito \\
\hline Divisão de Gestão da & $4.3,4.9,4.10 ; 4.11 ;$ \\
Qualidade & $4.12 ; 4.13 ; 4.14 ; 4.15$ \\
\hline Divisão de & 4.6 \\
Suprimentos & \\
\hline Setor de Serviços & $4.4,4.7,4.8$ e 5.10 \\
Técnicos & \\
Especializados & \\
\hline Divisão de Inovação e \\
Prospecção \\
Tecnológicas & Parte do item 4.3 \\
\hline
\end{tabular}

Continuando o processo, foram elaborados as Normas Gerenciais da Qualidade para os requisitos de Garantia da Qualidade (item 5.9), Manuseio de Itens de Ensaio (item 5.8) e Equipamentos (item 5.5) e, apresentados na reunião de análise crítica do Sistema de Gestão da Qualidade em 2010 aos Responsáveis Técnicos dos escopos acreditados e demais participantes da Reunião de Análise Crítica de documentação. Nesta reunião somente o documento referente à garantia da qualidade foi discutido e aprovado, ficando os outros para uma segunda etapa.
A DIGQ revisou os procedimentos restantes e os apresentou-os novamente na reunião de análise crítica do SGQ em março de 2011, na qual os mesmos foram aprovados. Em outubro de 2011, toda a documentação referente à unificação já estava implantada.

Em 2012, como uma das etapas para a consolidação da unificação foi solicitado ao INMETRO uma reunião que houvesse um entendimento por parte do mesmo sobre a unificação do sistema do INT durante a avaliação geral de todo o escopo do INT e de seu Sistema de Gestão da Qualidade único agendada para junho de 2012. Nesta oportunidade estava prevista a avaliação de parte do escopo do INT onde procuramos aproveitar para que fosse avaliado o escopo em sua totalidade e, criar o marco zero da unificação.

Foi após esta avaliação que o INT foi reconhecido como Sistema Único, mantendo como seu número de acreditação o CRL 0006, o primeiro conseguido por um laboratório do INT.

A motivação para a realização da unificação teve início em uma demanda do INMETRO, a qual foi aceita internamente, já que os laboratórios passariam a se dedicar mais com as atividades técnicas (ensaios) e somente uma área seria responsável pela Gestão da Qualidade. Além disso, os custos que envolvem a acreditação tenderiam a diminuir. Outro ponto importante, seria a otimização de novas acreditações, já que o laboratório se dedicaria somente a validação de seus ensaios.

De acordo com SANTOS (2011), adotar um sistema de gestão unificado significa utilizar sistemática única para a gestão, como, por exemplo, realização de análise crítica e auditorias internas da qualidade e registros de ações corretivas e preventivas. Essa opção reduz a quantidade de documentação, uma vez que há um único Manual da Qualidade e todos utilizam os mesmos procedimentos sistêmicos. A implantação desse sistema, de forma geral, também, agiliza e reduz os custos dos processos de acreditação. No entanto, esse tipo de sistema necessita de atenção especial para garantir a eficácia e a eficiência do Sistema de Gestão em todos os laboratórios que o compõe, porque as dificuldades para o monitoramento e controle do sistema podem ser potencializadas. 


\section{RESULTADOS E DISCUSSÃO:}

A implantação e manutenção de um Sistema de Gestão da Qualidade Unificado, eficaz e eficiente e em consonância com as metas institucionais e do Governo Federal, trouxe pontos positivos que já puderam ser sentidos, os quais estão listados abaixo:

- Gerenciamento único de documentos, sem ocorrência de duplicidade dos mesmos;

- Diminuição das atividades do corpo técnico do INT em atividades de gestão e, economia homem/hora, considerando que a falta de pessoal é uma realidade na instituição;

- Envolvimento da Alta Direção do INT no processo de acreditação;

- Redução de custos na realização de treinamentos envolvendo a norma ABNT NBR ISO/IEC 17025:2005;

- Facilidade de inclusão de escopos, através de extensões e não de novas acreditações;

- Realização de uma única auditoria interna referente ao item 4 abrangendo todo o escopo do INT;

- Gerenciamento único de calibrações;

- Realização de auditorias internas técnicas com a utilização do cadastro de auditores internos, não havendo conflito de interesse das partes envolvidas;

- Com a unificação, diversos controles puderam ser informatizados, o que possibilitou a disponibilização da informação em tempo real;

- Esforço menor na manutenção das acreditações;

- Mudança da cultura da qualidade do INT.
- Desde 2008, o Sistema de Gestão da Qualidade do INT foi alvo de 5 (cinco) avaliações por parte do INMETRO. No entanto, a descontinuidade dos avaliadores líderes para acompanhar o processo dificultou o entendimento da proposta da unificação apresentada pelo INT.

Os resultados quantitativos do último ano (2013) e as metas propostas para 2014 podem ser verificados no documento apresentado na Figura 1, analisado durante as Reuniões Anuais de Análise Crítica do Sistema de Gestão da Qualidade.

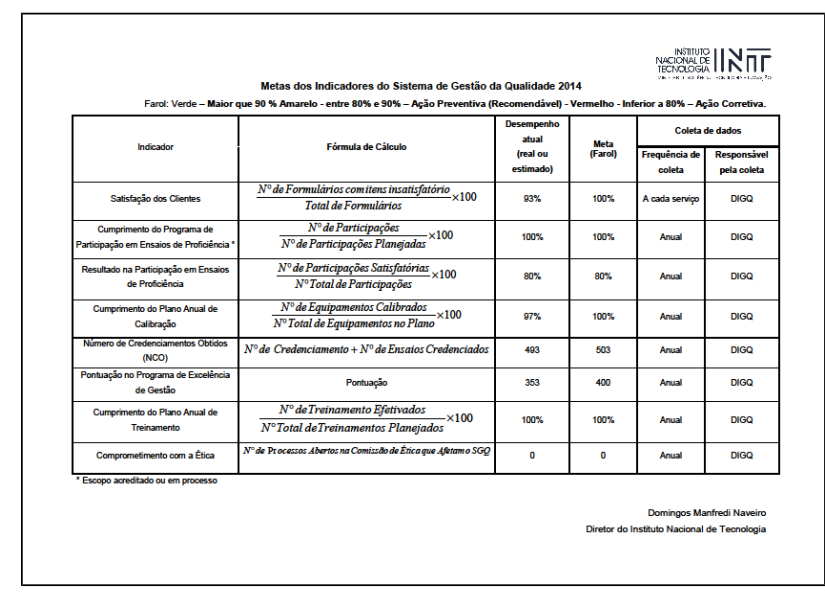

Figura 1. Meta dos Indicadores do Sistema de Gestão da Qualidade 2014

A Figura 2 apresenta do Logo de Acreditação do Instituto Nacional de Tecnologia INT na Rede Brasileira de Laboratórios de Ensaio - RBLE. O escopo de ensaios acreditados pode ser verificado através do link:

http://www.inmetro.gov.br/laboratorios/rble/detalh e_laboratorio.asp?nom_apelido=INT

\section{CONCLUSÕES:}

Apesar de todo o esforço do INT, pode ser citado como ponto negativo que a unificação demorou a ser consolidada pelo INMETRO, em virtude das seguintes dificuldades encontradas:

- $\quad$ Falta de entendimento por parte do INMETRO ao continuar realizando avaliações por laboratórios, como se os mesmos fossem ainda sistemas independentes;

PERIÓDICO TCHÊ QUÍMICA • www.periodico.tchequimica.com • Vol. 12 N. 23 - ISSN 1806-0374 (impresso) • ISSN 1806-9827 (CD-ROM) • ISSN 2179-0302 (meio eletrônico) 


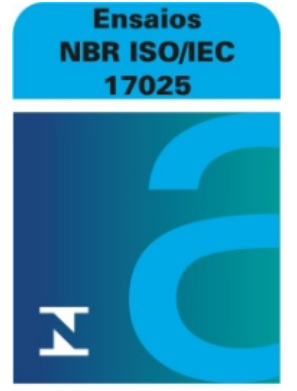

CRL 0006

rios do INT", ENQUALAB 2006, São Paulo, 2006.

6. Planejamento Estratégico do INT 2011 2021.

7. Regimento Interno do INT - Portaria MCT $n^{\circ}$ 762 de 28/11/2007, publicada no DOU de 29/11/2007.

8. Designação da Gerência da Qualidade das UOs laboratoriais do INT - Portaria INT $\mathrm{n}^{\circ}$ 031 de 12/05/2008.

Figura 2- Logo de Acreditação do Instituto Nacional de Tecnologia

Deverá apresentar, resumidamente, os dados discutidos em Resultados e Discussão mostrando a relevância do trabalho e quão diferente ou inovador a pesquisa é frente a outros trabalhos já publicados. Ainda, deverão ser destacados os benefícios e melhorias que podem ser observados para o desenvolvimento de novos padrões científicos que possam mudar algo na área relacionada.

\section{AGRADECIMENTOS:}

A todo corpo funcional do INT envolvido com a realização de ensaios acreditados e, a Direção do Instituto por tornar este trabalho possível.

\section{REFERÊNCIAS:}

1. ABNT NBR ISO/IEC 17025:2005, Requisitos gerais para a competência de laboratórios de ensaio e calibração.

2. L. L dos Santos, "Qualidade em Laboratórios de Ensaio e Calibração: Fatores Críticos e Propostas de Ações para Implementação do Sistema de Gestão" - Dissertação de mestrado, UFF, Niterói, 2011.

3. J. P. de Cerqueira, M. C. Martins, "Auditorias de Sistemas de Gestão: ISO 9001, ISO 14001, OHSAS 18001, ISO/IEC 17025, SA 8000, ISO 19011:2002", Rio de Janeiro: Qualitymark, 2004.

4. H. J. Clarim, M. G. P. A. Simões, I. M.V. Caminha, A. Travalloni, "Implantação de Sistema de Gestão da Qualidade em Laboratórios", V Metrosul, Curitiba, Paraná, 2007.

5. H. J. Clarim, M. G. P. A. Simões, I. M.V. Caminha, A. Travalloni, "Implantação de Sistema de Gestão da Qualidade nos Laborató- 